\title{
Le renouvellement des économies périphériques
}

\author{
Serge Côté et Marc-Urbain Proulx ${ }^{1}$
}

Le destin économique des divers territoires périphériques du Québec n'est pas tout tracé d'avance. Et ce destin n'est pas systématiquement conditionné par les « vocations naturelles» de ces territoires. Voilà deux des principales conclusions de notre étude livrée en 2002 à Développement Économique Canada (DEC). De nombreux phénomènes intéressants furent aussi constatés, permettant d'éclairer davantage une situation non métropolitaine fort complexe et difficilement saisissable par les modèles classiques d'analyse spatiale.

Dans les faits, les immenses régions périphériques du Québec représentent à l'évidence une collection de petits territoires de production, généralement organisés autour de leur propre petit centre d'extraction de ressources naturelles. Plusieurs centaines de ces centres sont ainsi dispersés sur le vaste espace, en fonction des bassins de ressources répartis très inégalement. Paradoxalement, ces économies territoriales exportatrices s'avèrent désenclavées vis-à-vis le marché international tout en étant relativement distantes et souvent même isolées dans l'ensemble spatial discontinu du Québec.

Si les économies territoriales sont fortement dépendantes des ressources naturelles, elles-mêmes soumises aux règles du marché mondial, nous avons illustré que la trajectoire historique et contemporaine de chaque région administrative apparaît bien distinctement dans son originalité. À l'exception de quelques poches ici et là telles que Matane, Cabano et Chandler, les territoires qui composent l'Est du Québec n'ont jamais connu de véritable décollage industriel alors que les conditions endogènes y étaient favorables, notamment dans le Bas-Saint-Laurent. Ce décollage ou démarrage fut cependant réel en Abitibi-Témiscamingue et au Saguenay - Lac-Saint-Jean, propulsé par des investissements exogènes massifs. Plusieurs territoires de la CôteNord ont aussi subi cette impulsion industrielle majeure, plus récemment cependant alors que le déclin ou la stagnation affectaient déjà de nombreux autres bassins périphériques en rupture de stocks. Notons aussi que la Beauce et les Bois-Francs ont connu un décollage industriel plus tardif que la plupart des régions périphériques.

\section{Économies territoriales immatures}

L'une des caractéristiques de l'évolution économique de ces territoires périphériques «décollés» réside dans leur passage direct à une phase de consommation de masse sans avoir vraiment traversé celle de la maturité économique associée à la transformation des ressources et à la diversification des activités industrielles. De fait, nos données saisies dans cette étude commanditée par DEC nous ont permis de visualiser une industrialisation encore infantile sur la Côte-Nord et en Gaspésie - Îles-de-la-Madeleine, pré-adolescente en Abitibi-Témiscamingue, plutôt adolescente dans le Bas-Saint-Laurent et post-adolescente au Saguenay - Lac-Saint-Jean, alors que plusieurs régions centrales ont atteint une phase post-adolescente (et même mature dans le cas de la Montérégie), à l'exception de l'Outaouais et de la Mauricie demeurées juvéniles. Cette immaturité économique, à degrés divers selon les régions, conditionne évidemment la structure de base sur laquelle les agents de développement souhaitent actuellement l'arrivée en régions non métropolitaines de ladite nouvelle économie ou du moins d'éléments de renouvellement économique.

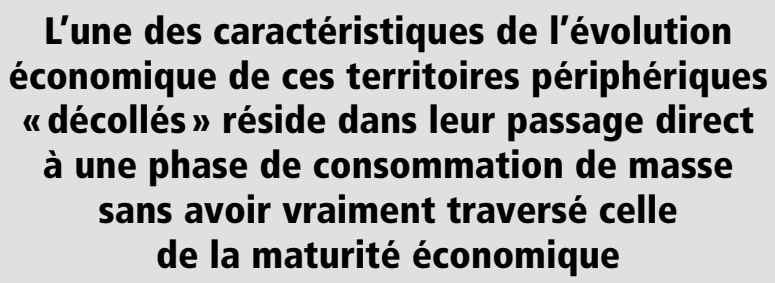

Nombreuses sont les causes de l'immaturité économique des régions périphériques. Parmi les principales, citons la très connue distance des grands marchés que sont les grandes régions métropolitaines de Montréal, Toronto ou New-York. Soulignons aussi les coûts élevés du 
travail qui certes ne représentent pas un facteur très attractif du secteur manufacturier. Entre autres causes, il y a la difficulté de créer et de retenir les savoir-faire détenus par une main-d'œuvre mobile par essence ainsi que la fuite constante des profits et de l'épargne hors des périphéries. Toutes ces causes d'immaturité pointées sont cependant difficilement mesurables d'une manière précise et donnent parfois lieu à des interprétations paradoxales. À titre d'exemple, si l'éloignement relativement moindre des grands marchés peut être évoqué comme facteur explicatif de la diversification industrielle comparativement beaucoup plus avancée dans le Bas-Saint-Laurent, en Basse-Mauricie et au Saguenay, des salaires plus bas et une économie traditionnelle plus forte ne semblent pas avoir été plus profitables au BasSaint-Laurent, à Charlevoix et en Outaouais dont les économies territoriales demeurent aujourd'hui insuffisamment diversifiées en regard de leur potentiel théorique.

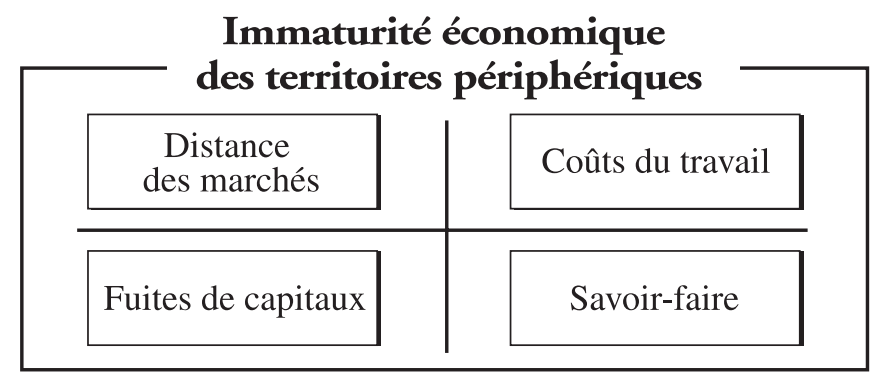

L'entrée plus ou moins rapide des territoires périphériques dans la phase de consommation de masse s'explique en partie par les hauts salaires versés dans la plupart des activités reliées à l'exploitation des ressources naturelles. Les investissements publics et la desserte universelle des services publics ont aussi participé à ce mouvement de consommation généralisée. Notons que cette consommation de biens et services distribués par des activités commerciales rapidement rentables, a par ailleurs monopolisé l'entrepreneuriat territorial issu largement du surplus de main-d'œuvre agricole et forestière libéré par la mécanisation dans ces secteurs après 1945. L'enclavement variablement bienfaiteur de ces territoires dispersés sur l'espace québécois a favorisé le cumul de capital autour d'une élite d'affaires confortée par ce protectionnisme spatial, la rendant vulnérable face à la concurrence. Car si plusieurs fabriques locales ont bénéficié à une certaine époque d'une protection par la distance, leurs marchés furent insuffisamment importants cependant pour permettre l'émergence et la rétention de grandes unités de production compétitives à l'échelle nationale.

De 1940 à 1970, les économies périphériques ont vécu des années glorieuses à la faveur d'une forte demande internationale de matières premières dans un contexte concurrentiel avantageux. Soutenues en outre par une importante vague d'investissements publics dans les infrastructures de transport et les équipements divers (santé, éducation, loisirs, communautaire, etc.), les économies territoriales étaient relativement prospères au début des années 1970. Malgré cette prospérité et ce cumul, nous avons noté que les économies territoriales périphériques étaient encore et toujours largement tributaires des capitaux extérieurs pour maintenir leurs investissements et leur croissance. Peu de valeur aux ressources naturelles fut ajoutée sur les lieux d'extraction, si ce n'est une première transformation.

\section{Changements structurels}

Depuis trois décennies, des changements structurels importants frappent les activités de production liées aux ressources dans les régions périphériques du Québec. Ces changements affectent habituellement le niveau de l'emploi. Deux cas de figure sont présents, en s'inscrivant dans un contexte ayant des caractéristiques particulières.

Dans le premier cas, les activités d'extraction et de transformation subissent l'arrivée de nouvelles technologies de production qui affectent variablement tous les secteurs par une forte addition de capitaux et une réduction des besoins de main-d'œuvre. La ressource naturelle continue à être exploitée intensivement, mais les entreprises procèdent à des rationalisations dictées par des changements technologiques majeurs ou par des restructurations d'envergure, souvent accompagnées de changements dans la propriété des entreprises. Constatons que, d'une manière générale, on effectue davantage de livraisons de matières brutes, mais on utilise moins de travailleurs pour les extraire. C'est-à-dire que le rapport vertueux entre la production et l'emploi ne fonctionne plus.

Dans le second cas de figure, la mise en valeur de la ressource entre en sommeil et s'accompagne en conséquence d'une diminution de la production et de l'emploi, soit à cause d'un manque de disponibilité de la matière première, soit à cause d'un changement dans les conditions du marché. Il existe de nombreux exemples de ce cas, notamment dans le fer, le poisson, le cuivre, l'élevage, le bois et l'agriculture. La professionnalisation du travail agricole et du travail en forêt a conduit par ailleurs à la réduction du nombre des agriculteurs et des forestiers et a amené une baisse radicale des petits producteurs pluriactifs.

Plusieurs territoires agricoles, forestiers, maritimes et miniers furent ainsi disqualifiés, entièrement ou en partie. Si bien que des zones entières subissent actuellement un 


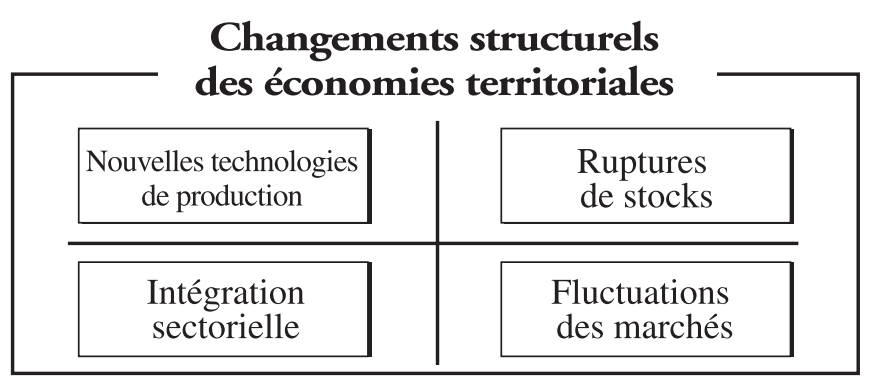

lent dépeuplement. Plusieurs centres d'extraction stagnent. L'économie de consommation de masse continue certes sa lancée (tertiarisation) comme ailleurs, mais à un rythme désormais plus lent, limitée notamment par un revenu moyen par ménage relativement décroissant. D'autres impacts spatiaux se manifestent, soit l'apparition de certaines poches de pauvreté, la dégradation du cadre bâti, la sousutilisation d'équipements publics et l'étalement urbain onéreux pour une base foncière à peu près stagnante.

\section{D'autres impacts spatiaux se manifestent, soit l'apparition de certaines poches de pau- vreté, la dégradation du cadre bâti, la sous- utilisation d'équipements publics et l'étalement urbain onéreux pour une base foncière à peu près stagnante.}

Quel que soit le cas de figure, tous ces impacts généraux semblent s'intensifier encore actuellement sous l'égide d'une grande vague d'intégration.

\section{Intégration sectorielle en toile de fond}

En effet, les territoires périphériques subissent comme les autres au Québec le mouvement universel d'intégration et de concentration sectorielles dans les domaines de la production et de la distribution. Le mouvement semble être soutenu non seulement par la nécessaire intensification du capital dans les activités économiques mais aussi par une plus forte mobilité des facteurs, notamment les travailleurs et les consommateurs. Les marchés étant limités en périphérie, il est d'abord fort difficile d'y maintenir d'importantes unités de production qui préfèrent se localiser à proximité des grands marchés, notamment dans le secteur agro-alimentaire qui voit se délocaliser ses entreprises traditionnelles de plus en plus intégrées désormais. Il est clair à notre lecture de la situation que la consolidation des activités de production standardisée (pain, vêtements, lait, meubles, etc.) au sein des unités gigantesques profite largement au sud-est du Québec. Les lieux où sont localisées les unités intégrées forment en réalité une longue zone industrielle polycentrique. Se dessine ainsi une sorte de croissant manufacturier qui s'appuie sur la ligne géogra- phique des Appalaches entre le nord de Montréal et le sud de Québec. La frontière américaine devient à l'évidence un facteur d'attraction industrielle en croissante importance dans le double contexte de l'ALENA et de l'intégration sectorielle de la production.

\section{Il est clair à notre lecture de la situation que la consolidation des activités de production standardisée (pain, vêtements, lait, meubles, etc.) au sein des unités gigantesques profite largement au sud-est du Québec.}

Dans les activités de distribution qui représentent au Québec le principal moteur de la concentration de la population et des services à notre époque, les succursales de chaînes nationales et internationales ont largement remplacé les commerces et services locaux dans l'alimentation, les vêtements, la quincaillerie, le meuble, la restauration, etc. L'actuelle arrivée des Géants de la distribution (WalMart; Tanguay; Loblaw; Réno Dépôt; Super C; etc.) ne fait qu'accentuer ce mouvement de concentration en ayant aussi des incidences spatiales très importantes puisque ces grandes surfaces s'implantent dans les grands centres régionaux de consommation tels que Rimouski, Baie-Comeau, Alma, Val-d'Or, Matane, Sept-Îles, etc., au détriment des plus petits qui se font drainer leurs consommateurs et en conséquence leurs commerces de petite et moyenne dimensions. On comprend ainsi que la plupart des rues principales des centres urbains sont largement dévitalisées au profit de nouvelles concentrations sur les grands boulevards péri-urbains qui accueillent les grandes surfaces, les centres commerciaux et les édifices à bureaux dont les occupants sont généralement des entreprises à propriété extrarégionale. Il faut aussi noter que ce mouvement se produit en régions périphériques dans un contexte de stagnation de la consommation causée par la décroissance démographique, la baisse relative du revenu moyen par ménage et le vieillissement de la population.

Bref, avec l'intensification capitalistique qui réduit la masse salariale versée dans les régions tout en intégrant plus fortement les activités économiques, les fuites de capitaux hors des circuits économiques territoriaux sont plus importantes qu'auparavant. Les conséquences se manifestent sous la forme de cercles vicieux plus ou moins apparents de décroissance territoriale. Quelques mécanismes de rétention tels que les Fonds communautaires, certaines caisses de retraite ainsi que les partenariats financiers tentent de colmater la brèche. Mais le flot est très difficile à combattre puisqu'il n'y a pas suffisamment de projets rentables pour utiliser ce capital en régions périphériques. 


\section{Deux bonne nouvelles}

Nous avons noté deux phénomènes qui s'inscrivent à contre tendance de toutes ces forces qui occasionnent des difficultés aux économies territoriales non métropolitaines du Québec. Soulignons d'abord la formation de petites grappes d'activités dans l'industrie secondaire et dans le tourisme. On pense par exemple à des filières industrielles embryonnaires dans des secteurs comme l'impression et le matériel de transport dans le BasSaint-Laurent, les ateliers d'usinage et les produits de l'aluminium au Saguenay - Lac-Saint-Jean, la construction et la réparation de navires en Gaspésie - Îles-de-laMadeleine. Ces filières en début de structuration contribuent à la diversification des traditionnelles économies territoriales périphériques, centrées sur le bois, l'agroalimentaire, la pêche et les mines. Il faut aussi mentionner que de petites filières de l'industrie touristique se forment ici et là autour des attractions majeures localisées à Percé, Mingan, Tadoussac, Val-Jalbert, Anse-Saint-Jean, Saint-Jean-Port-Joli... Ces petits centres touristiques dispersés sur le vaste espace s'ajoutent au nombre de centres d'extraction plus traditionnels de ressources naturelles. Il est intéressant de noter que toutes ces activités traditionnelles ou non traditionnelles s'avèrent relativement parsemées sur la surface des régions périphériques.

Le second phénomène digne de mention concerne l'émergence d'initiatives innovatrices qui prennent souvent la forme de spécialités ou de niches. Il peut s'agir de productions liées aux ressources, comme les produits de l'agriculture biologique, l'aquaculture, l'extraction des protéines, ou de chitosane, à partir de résidus marins ou encore le bois de structure à haute résistance; ou il peut s'agir de productions indépendantes des ressources comme la fibre optique, le verre trempé, la machinerie spécialisée, les composantes de précision, les contrôles électroniques, les jeux vidéo, etc. Dans le domaine des services, des spécialisations ont aussi surgi et caractérisent désormais certaines entreprises: logiciels dédiés, formation à distance, multimédia, centres d'appel. Ces activités émergentes, qui ne sont pas toujours décelables dans les statistiques globales, représentent néanmoins un potentiel non négligeable de renouvellement des économies régionales périphériques. Dans tous les cas, les produits en cause sont bien typés, requièrent une maîtrise poussée des procédés, se distinguent par la qualité de leur exécution et s'adressent à des clients exigeants souvent situés à l'extérieur de la région.

Plusieurs petits centres ruraux bénéficient de la localisation de ces productions innovatrices. Plus d'une centaine d'entre eux sont en croissance démographique et s'affir- ment dans leur rôle de rétention de la population et de consolidation de la structure de peuplement rural. Ils maintiennent des services de proximité et attirent de nouvelles activités économiques. Notons finalement, que les principaux pôles régionaux jouent un rôle économique important en concentrant non seulement les activités de distribution - consommation mais aussi les services supérieurs qui leur permettent d'assumer un leadership économique considérable.

\section{Nos recommandations}

Il serait dommage de terminer cette synthèse sur le diagnostic des économies périphériques du Québec sans rappeler au lecteur que de nombreuses ressources naturelles demeurent curieusement sous-exploitées malgré la sur-exploitation évidente en de nombreux lieux. Avec 3\% des réserves planétaires, l'eau douce représente une ressource aux nombreux potentiels. Dans nos 75 millions d'hectares de forêts exploitables, le reboisement et le jardinage n'ont pas encore atteint le niveau adéquat d'une ressource qui serait en renouvellement optimal. Dans l'agriculture, de très nombreuses terres insuffisamment productives pour une mise en valeur intensive, demeurent malheureusement peu utilisées pour des productions différentes. Dans les ressources maritimes, le fleuve et le golfe Saint-Laurent demeurent encore en dessous de leur capacité de production dans un contexte de mariculture éventuellement régulée. Le Québec périphérique possède aussi un potentiel hydraulique et éolien lui permettant de doubler éventuellement la production actuelle d'énergie. Et que dire des vastes étendues nordiques à explorer à l'aide des nouvelles techniques de prospection des ressources minérales exploitables? Notons aussi que les divers territoires qui composent la périphérie du Québec, aussi immense soit-elle, sont généralement bien nantis en infrastructures, en équipements et en services publics. Ils possèdent alors les moyens physiques pour supporter des ajouts de savoir-faire, de technologie et de capitaux afin d'améliorer leur productivité et leur production de richesses.

Notre première recommandation concerne la revalorisation des ressources naturelles actuellement sous-utilisées par les activités industrielles fondées sur une mécanisation intensive et une production massive. Des usages de remplacement généralement (pas toujours) moins mécanisés mais plus intenses en savoir-faire doivent devenir le cœur d'une stratégie gouvernementale explicite. Nous pensons notamment à la transformation des aliments, du bois et des métaux, à l'aménagement des forêts, aux applications des technologies numériques dans les domaines de l'instrumentation et des équipements marins, des transports, de l'énergie, des communications, du divertissement. Il faut en ce sens encourager la distinction des produits fabriqués afin que 
leur positionnement sur le marché puisse trouver chacun sa niche spécifique à travers les produits standardisés fabriqués en très grande série.

L'innovation doit à cet effet devenir un mode de vie des travailleurs. La stratégie de savoir-faire pourrait être appliquée par diverses mesures publiques. Le transfert de savoirs dans les domaines de la biomasse, de l'énergie éolienne, de la métallurgie, des communications, de la foresterie, des ressources minérales, de l'agriculture, etc., devrait alors s'inscrire comme une simple activité courante. La formation continue des opérateurs actuels et en émergence serait appropriée pour ancrer et renouveler les expertises et les savoir-faire. Des agents de R\&D mobiles pourraient cibler leurs actions sur les petites unités de production dispersées sur les territoires, ce qui ne les empêcherait pas, bien au contraire, de travailler en réseau avec les autres agents. Le dépistage de savoir-faire serait effectué à l'étranger afin de favoriser la pratique très courante de l'innovation par imitation et diffusion.

\section{L'innovation doit à cet effet devenir un mode de vie des travailleurs.}

Nous proposons aussi dans notre rapport livré à DEC en 2002, l'utilisation du concept de «filière de production» pour saisir et formaliser les processus de valorisation en chaîne des ressources naturelles par des productions innovatrices intégrées, afin de cibler davantage les segments qui apparaissent porteurs, notamment sous l'angle de la $\mathrm{R} \& \mathrm{D}$, du transfert vertical de technologies et de la formation des ressources humaines. Si les principaux pôles régionaux doivent certes être davantage mis à contribution, les petits centres ruraux de services dont plusieurs polarisent actuellement l'emploi et les activités devraient être l'objet d'une attention particulière dans cet esprit de structuration de filières, d'émancipation des savoir-faire et de mise en marché de produits ou de services distinctifs.

Notre deuxième recommandation concerne la planification de Systèmes territoriaux d'innovation et de production (STIP), soit spécialisés dans un secteur, soit orientés vers la diversification. Nous pensons notamment à la nouvelle zone polycentrique de production diversifiée (croissant manufacturier), localisée au sudest du Québec. Au-delà des découpages administratifs, cette vaste aire manufacturière en émergence doit être dotée d'une vision globale, de stratégies explicites et d'un plan d'actions concrètes, notamment à l'égard du système de transport. Nous pensons aussi particulièrement à la technopôle maritime qui touche plusieurs régions de l'Est du Québec et au district industriel embryonnaire du Saguenay appelé Vallée de l'aluminium. Dans ces deux cas de territoires périphériques relativement diversifiés, leur spécialisation souhaitable dans ces deux domaines clés ne doit pas mettre en péril leur processus de diversification en cours. Aussi, plusieurs territoires, tels que ceux sur lesquels oeuvrent les SADC (sociétés d'aide au développement des collectivités), les CLD (centres locaux de développement) et autres centres de services au développement, ont tout intérêt à se doter d'un tel concept (STIP) intégrateur des diverses interventions selon leur vocation.

Une stratégie gouvernementale STIP permettrait alors de mettre en place les conditions de la coordination des diverses interventions à l'égard de la $\mathrm{R} \& \mathrm{D}$, de la localisation d'unités de recherche (centres, groupes, laboratoires), du dépistage de technologies, de la mise en place d'équipements publics, de l'attraction d'activités économiques, de la qualification des ressources humaines, du soutien financier au démarrage ou à la croissance, de l'animation socio-économique, du réseautage, etc. Une telle stratégie contribuerait à relever les défis de la flexibilité du travail, du transfert de savoir-faire, du financement des initiatives et de la circulation de l'information. Étant donné le nombre et la diversité des acteurs, une procédure appropriée de planification territoriale demeure la meilleure solution institutionnelle. À la lumière de l'expérience acquise dans les pays de l'OCDE, nous proposons à la planification territoriale un objectif à l'égard de la mise en place de «conventions territoriales» d'innovation et de production permettant de commettre non seulement les secteurs public et privé mais aussi la société civile organisée, y compris les syndicats, dans des processus d'apprentissage collectif ancrés sur les territoires.

\section{Une telle stratégie contribuerait à relever les défis de la flexibilité du travail, du transfert de savoir-faire, du financement des initiatives et de la circulation de l'information.}

Les économies régionales périphériques du Québec sont en train de changer considérablement sous nos yeux. Certains des changements qui surviennent, particulièrement ceux qui se traduisent par des pertes d'emplois, freinent le développement. D'autres conduisent à un renouvellement des tissus économiques régionaux. Ce renouveau s'appuie dans tous les cas sur l'innovation, qui apparaît comme le facteur clé du développement économique actuel et à venir. Les recommandations qui viennent d'être formulées visent en somme à favoriser la mise en œuvre des innovations et à stimuler le développement des régions.

\section{Note et référence}

1 Serge Côté est professeur à l'Université du Québec à Rimouski et directeur du GRIDEQ. Marc-Urbain Proulx est professeur à l'Université du Québec à Chicoutimi et directeur du Doctorat UQAR-UQAC en développement régional. 